\title{
Data Curation Practices in Institutional Repositories: An Exploratory Study
}

\section{Abstract}

This poster reports on the preliminary findings of a study that examines data curation work in institutional repositories (IR). The study identified three perceived roles played by IR staff (i.e., data curator, IR manager, and metadata specialist) and role-specific sets of activities and skills. The findings of this study can inform the development of best practices and effective infrastructure support for data curation in the context of IRs, as well as teaching data curation in LIS schools.

\section{Research Questions}

- What are the typical data curationrelated activities and contexts in IRs?

- How does IR staff perceive their rolerelated activities and skills?

- What user services do IRs currently support?

\section{Methodology}

The study used Activity Theory (Engestrom, 1987; Leontiev, 1978) to guide the design of a protocol for semistructured interviews. Activity Theory suggests general activity structure and context relationships of data curation in IRs.

This poster reports on data collected from five participants from five different universities in the US. The selection of participants was guided by two criteria. To be eligible for participation in the study, participants had to work for IRs that stored and curated research data objects and had to be operated by AAU member universities.

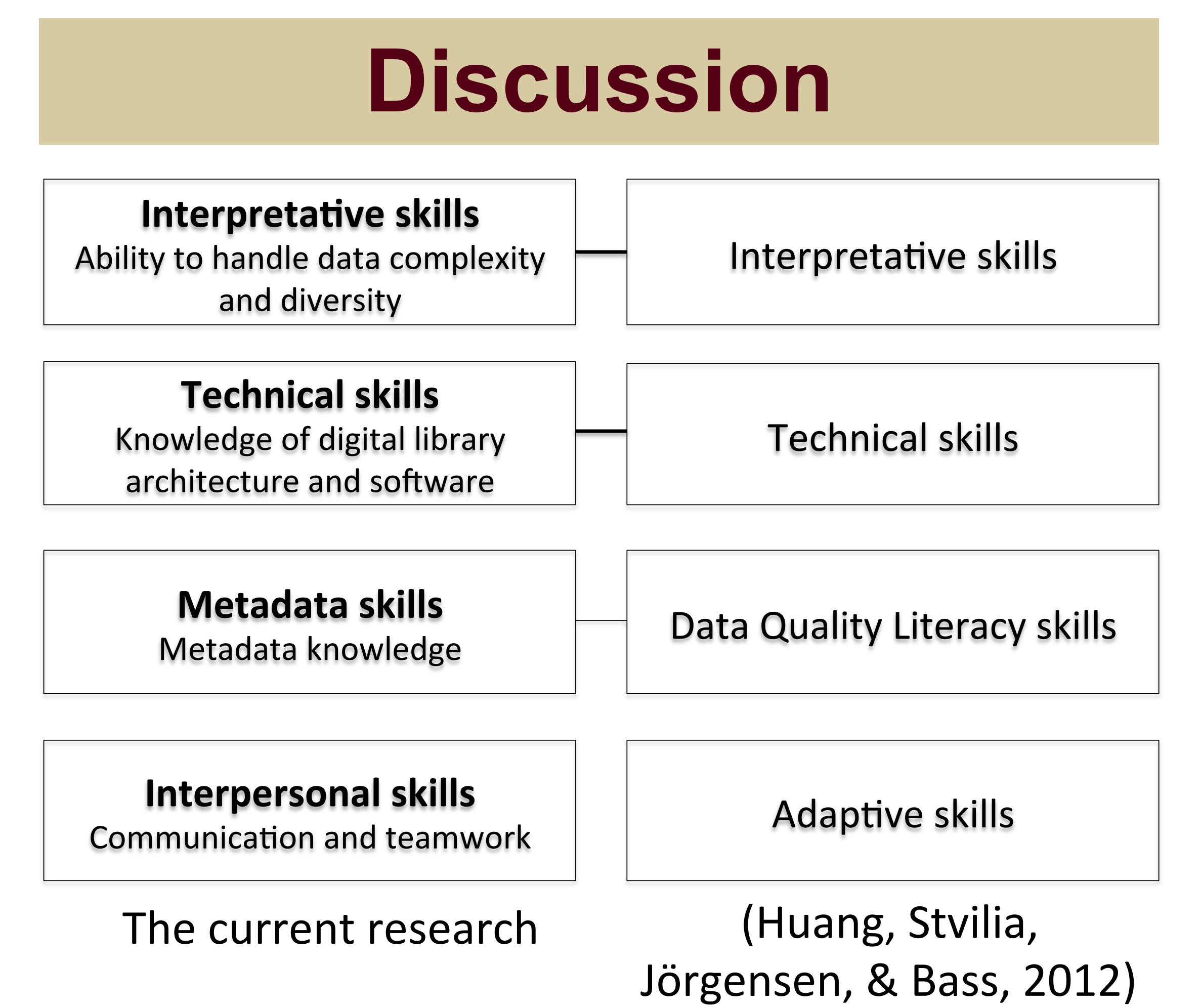

\section{Data Curation Activities and Contexts}

\begin{tabular}{|c|c|c|c|c|c|}
\hline \multicolumn{6}{|c|}{ Activities: } \\
\hline $\begin{array}{l}\text { - Conceptualize } \\
\text { data with } \\
\text { data } \\
\text { providers }\end{array}$ & $\begin{array}{l}\text { - Receive data } \\
\text { and create } \\
\text { metadata }\end{array}$ & $\begin{array}{l}\text { - Evaluate data } \\
\text { for } \\
\text { preservation }\end{array}$ & $\begin{array}{l}\text { - Ingest and } \\
\text { store data to } \\
\text { repository }\end{array}$ & - Publish data & $\begin{array}{l}\text { - Ensure that } \\
\text { data is } \\
\text { accessible and } \\
\text { reusable }\end{array}$ \\
\hline \multicolumn{6}{|c|}{ Tools: } \\
\hline \multirow{2}{*}{\multicolumn{6}{|c|}{$\begin{array}{c}\text { •Software (e.g., word, excel, powerpoint, photoshop, video compression tool, image viewer) } \\
\text {-Internet web application (e.g., email, Altmetrics, file recommendataion/identification tool, EZID service, } \\
\text { Google Refine and Drive) }\end{array}$}} \\
\hline & & & & & \\
\hline \multicolumn{6}{|c|}{-Metadata schemas (e.g., Doublin Core, Premis, DataCite, MODS, METS, MIX) } \\
\hline \multicolumn{6}{|c|}{-Identifier schemas (e.g., DOI, Handle, ARK) } \\
\hline \multicolumn{6}{|c|}{ - Repository software (e.g., DSpace, bepress Digital Commons, HUBzero, Hydra) } \\
\hline \multicolumn{6}{|c|}{-High capacity storage } \\
\hline \multicolumn{6}{|c|}{-Workflow add-on to IR system } \\
\hline \multicolumn{6}{|c|}{ Norms and Rules: } \\
\hline \multicolumn{6}{|c|}{ - For deposit, content, digital preservation, copyright, collection, support, access, and use } \\
\hline \multicolumn{6}{|c|}{ Division of Labor: } \\
\hline \multicolumn{6}{|c|}{ IR manager, curator, metadata specialist, data provider } \\
\hline
\end{tabular}

\section{IR Staff's Role-Related Activities and Skills}

\begin{tabular}{|c|c|c|c|}
\hline & Data Curators & IR Managers & Metadata Specialists \\
\hline \multirow[t]{2}{*}{ 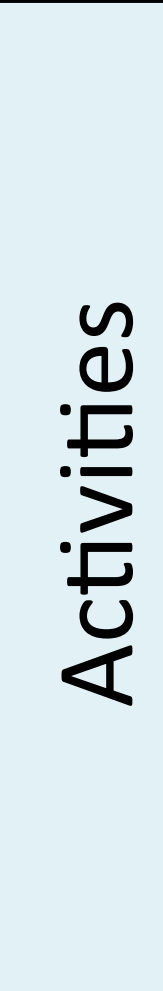 } & $\begin{array}{l}\text { - Build or plan data governance } \\
\text { structures } \\
\text { - Consult with data providers } \\
\text { and connect them to } \\
\text { metadata specialists or IR } \\
\text { managers }\end{array}$ & $\begin{array}{l}\text { - Manage IRs on a daily basis } \\
\text { Work with data providers to } \\
\text { help add metadata and upload } \\
\text { data into IRs }\end{array}$ & \multirow[t]{2}{*}{$\begin{array}{l}\text { - Help data providers to create } \\
\text { appropriate metadata for } \\
\text { their dataset }\end{array}$} \\
\hline & \multicolumn{2}{|c|}{ - $\quad$ Outreach and educate campus community } & \\
\hline \multirow{2}{*}{$\frac{n}{\frac{\bar{n}}{n}}$} & $\begin{array}{l}\text { - Familiarity with research data } \\
\text { (e.g., ability to handle data } \\
\text { complexity and diversity) }\end{array}$ & $\begin{array}{l}\text { - Technical skills (e.g., } \\
\text { knowledge of digital library } \\
\text { architecture and software) }\end{array}$ & - Metadata knowledge \\
\hline & \multicolumn{3}{|c|}{$\begin{array}{c}\text { - Ability to communicate well with other people and to work in a team } \\
\text { Time management }\end{array}$} \\
\hline
\end{tabular}

\section{IR User Activities and Services}

\begin{tabular}{|l|l|}
\hline \multicolumn{1}{|c|}{ IR User Activities } & \multicolumn{1}{c|}{ User Services } \\
\hline - Searching & - Providing options of using a license \\
- Browsing & - Providing usage statistics services \\
- Social networking/Sharing/Tagging & - Running data curation workflow wizard \\
- Citing & - Supporting project management functions \\
- Storing & \\
- Downloading & \\
\hline
\end{tabular}

\section{Next Steps}

Studying data curation work in IRs, identifying different roles played by IR staff and rolespecific needs for skills, can inform the development of best practices, infrastructure configuration templates, as well as the teaching of data curation in LIS schools. The future research related to this one will explore metadata needs and practices, particularly identifier needs and uses in the context of data curation in IRs.

$7^{\text {TH }}$ ASIS \& T ANNUAL MEETING October 31 - November 4,2014 Sheraton Seattle Hotel, Seathe, WA
Dong Joon Lee, dl10e@my.fsu.edu Besiki Stvilia,bstvilia@fsu.edu

Florida State University 